\title{
Application of an Electronic Tongue towards the analysis of brandies
}

\author{
Xavier Cetó $^{1}$, Matias Llobet ${ }^{2}$, Joan Marco $^{2}$ and Manel del Valle ${ }^{1, *}$ \\ ${ }^{1}$ Sensors and Biosensors Group, Department of Chemistry, Universitat Autònoma de \\ Barcelona, Edifici Cn, 08193 Bellaterra, Barcelona, SPAIN \\ ${ }^{2}$ Miguel Torres SA, Vilafranca del Penedès, Barcelona, SPAIN
}

\begin{abstract}
This work reports the application of a voltammetric Electronic Tongue (ET) in the analysis of brandies, specifically in its classification according to the scores given by a skilled sensory panel and in the discrimination of different ageing methods. For this purpose, spirits were analyzed with no other pretreatment than its dilution with a saline solution to ensure enough conductivity. Recorded voltammetric signals produced by an array of six modified epoxy-composite sensors were preprocessed employing Fast Fourier Transform in order to reduce the complexity of the input signals while preserving the relevant information. Then, using the obtained coefficients, responses were evaluated using Linear Discriminant Analysis (LDA) as the pattern recognition model used to carry out the classification tasks. In both cases, good prediction ability was attained by the ET (classification rates of $100 \%$ and $97 \%$, respectively), therefore permitting the correct classification of the different samples under study. Furthermore, two Artificial Neural Network models were also trained for the semi-quantitative identification of some undesired compounds markers of some brandy defects upper certain levels (namely butan-2-ol, ethyl acetate, acetaldehyde and butan-1-ol; r>0.975) and the quantification of polyphenol index $\mathrm{I}_{280}(\mathrm{r}=0.977)$.
\end{abstract}

Keywords: Electronic Tongue; voltammetric sensors; classification; taste; ageing; brandy

\footnotetext{
*E-mail: manel.delvalle@uab.cat; tel: +34 93 5811017; fax: +34 935812477

$\dagger$ Electronic Supplementary Information (ESI) available: Tables containing detailed information of the samples under study and examples of the recorded voltammograms for the whole sensor array and both subsets.
} 


\section{Introduction}

Over the last years, the use of Electronic Tongues (ETs) is arising as a promising approach to analyze liquid samples ${ }^{1,2}$. Such analytical systems are formed by an array of sensors where several sensing units, which exhibit different responses to various compounds, are coupled with advanced signal processing methods based on pattern recognition or multivariate response models, which allow for the qualitative or quantitative analysis of different sample parameters ${ }^{3}$.

In essence, ETs mimic the sense of taste in mammals, where a few receptors can respond to a large variety of substances in a combinatorial approach; this sensing principle is next coupled with a complex data treatment stage, as it is done in the brain, allowing to quantify or to classify perceptions or substances. These biomimetic systems, then in contrast to ever more selective classical methods, assume the combination of low selectivity sensors arrays (or with cross response features) in order to obtain some added value in the generation of analytical information.

Brandy is a spirit produced from wine distillates finally containing ca. $36 \%-45 \%$ alcohol by volume (abv) ${ }^{4}$. The production of brandy is a complex process that consists of several major steps including the production of base wine. Among brandies, the most well-known is Cognac; although this refers only to a type of brandy made in that region of France, many producers from different countries try to emulate its production methods. In particular, brandy must be made from specific grape varieties, and it must be twice distilled in copper pot stills and aged at least two years in French oak barrels from certain regions. Brandy matures in the same way as whisky and wine when aged in barrels, and most brandies are aged considerably longer than the minimum legal requirement to improve its sensorial features. Usually, before blending, the aged brandy is first tasted by a panel to be classified and to asses its quality.

Classification of brandies is usually performed by a skilled sensory panel, given the difficulties to perform this assessment using classical analytical techniques. That is, the main compounds primarily responsible for the style of brandy have not been clearly identified yet, what in turn hinders the use of classical methods which would imply identifying the compounds responsible of those sensations. On the contrary, ETs combine all the extracted chemical information from the array of sensors to generate a model like the human brain would do, avoiding to focus on which specific compounds are the responsible for those flavours or tastes; but also recognizing a specific 
fingerprint to identify or quantify them. A correctly trained ET would extract knowledge from the expert panel, and apply it in correspondence to the sensor's available information to reproduce operation of the former. Hence, the obtaining of an automated method would allow to significantly reduce brandy tasting by the panel, not to replace it, and to increase the number or frequency of samples that can be analyzed. In this way, ETs may provide a helpful tool that might be used as screening method either to asses producers guarantee quality control standards or to detect any adulteration or contamination.

Within the context, ETs have already been successfully applied to the classification of several alcoholic beverages such as wines ${ }^{5}$, cava wines ${ }^{6}$, beers ${ }^{7,8}$, whiskies ${ }^{9}$ or even sakes ${ }^{10}$; besides, ETs have been able to focus in some cases on the detection of inappropriate handling practices ${ }^{11}$, the use of alternative ageing methods ${ }^{12}$ or the adulteration of samples ${ }^{9}$. A unique prior attempt of application of ETs to qualify brandies is a conference communication from Legin's group in Saint Petersburg ${ }^{13}$; also classification of some eau-de-vie samples was attempted by the same group ${ }^{14}$.

The main goal of this work was the application of an ET that would allow the recognition and discrimination of the different types of brandies, in a similar way a sensory panel would do, allowing also the detection of adulterations in the production process. To this end, samples were measured using an array of modified epoxy-graphite voltammetric sensors; then, their complex voltammetric responses were preprocessed employing FFT in order to extract the significant information and to compress the departure information, next evaluating its response with a pattern recognition method to discriminate the different samples. In addition, two different ANN models were also trained to semi-quantitatively predict the concentration of some undesired compounds which are markers of some defects of brandies upper certain levels and to quantitatively assess their polyphenol content.

\section{Experimental}

\subsection{Reagents and solutions}

All reagents used were analytical reagent grade and all solutions were prepared using deionised water from a Milli-Q system (Millipore, Billerica, MA, USA). Copper, platinum and silver nanoparticles $(<50 \mathrm{~nm})$, cobalt (II) phtalocyanine and polypyrrole 
were purchased from Sigma-Aldrich (St. Louis, MO, USA). $\mathrm{KCl}$ was purchased from Merck KGaA (Darmstadt, Germany).

\subsection{Samples under study}

A total set of 74 wine spirits samples were analyzed. These samples were divided into two different subsets in order to evaluate the capabilities of the ET in the qualitative analysis of brandies from two different points of view. In the first case, samples considered were selected considering their taste attributes; while in the second, the different methods used in the ageing of brandy were studied. Detailed information of the samples under study could be found in supplementary Tables S1 and S2.

\subsubsection{Taste attributes}

A total of 41 samples, previously selected and tasted by a sensory panel, were considered. Those samples were classified into three different groups by the sensory panel according to its taste attributes; in this manner, most appreciated samples were assigned to category "taste 1", while worst ones were assigned to "taste 3". Hence, in this case the objective was to evaluate if the ET was able to classify the samples in the same manner the sensory panel would do.

\subsubsection{Ageing of the brandy}

Additionally, another set of 33 samples, all of them from the 2009 vintage (except the ones aged in new barrel), were analyzed. In this case, samples were selected in order to obtain a set of samples that have been aged using different methods; i.e. the use of pieces of wood (chips, sticks, blocks and strips) in the ageing of brandy to resemble its ageing in new barrels while accelerating the process. Thus, the aim was to asses if the ET is capable of distinguishing between the different ageing methods.

To ensure that discrimination was due to the different ageing method, two precautions were taken into account. Firstly, all samples considered were from the 2009 vintage. Additionally, polyphenol index $\mathrm{I}_{280}$ was also measured to confirm that no trend in its value was found between different methods. Hence, to ensure that those parameters are not the source of the discrimination between samples by the ET. 


\subsection{Electronic Tongue}

\subsubsection{Sensor array}

Based on previous studies in our laboratory ${ }^{6,11,15}$, an array of 6 voltammetric electrodes were prepared following the conventional methodology in our research group 16. First, a resin EpoTek H77 (Epoxy Technology, Billerica, MA, USA) and its corresponding hardener compound were mixed in the ratio $20: 3(\mathrm{w} / \mathrm{w})$; after that a $15 \%$ of graphite $(\mathrm{w} / \mathrm{w})$ and a $2 \%$ of the modifier $(\mathrm{w} / \mathrm{w})$ were added to the previous mixture before hardening, obtaining the composite. Then, it was manually homogenized for 60 min, and afterwards, the paste was allowed to harden for 3 days at $80^{\circ} \mathrm{C}$. Finally, the electrode surface was polished with different sandpapers of decreasing grain size, with a final electrode area of $28 \mathrm{~mm}^{2}$.

In this manner, one blank electrode plus 5 different bulk-modified voltammetric sensors were prepared using bare graphite $\mathrm{C}$ in the first case, and adding different modifiers such as copper, platinum and silver nanoparticles, cobalt phtalocyanine and polypyrrole to the bulk mixture - one component per electrode.

\subsubsection{Apparatus and measurement cell}

The amperometric measurement cell was formed by the 6-sensor voltammetric array plus a commercial platinum counter electrode (Model 52-67, Crison Instruments, Barcelona, Spain) and a reference double junction $\mathrm{Ag} / \mathrm{AgCl}$ electrode (Thermo Orion 900200, Beverly, MA, USA). Cyclic Voltammetry measurements were taken using a 6-channel AUTOLAB PGSTAT20 (Ecochemie, Netherlands), in a multichannel configuration, controlled with GPES Multichannel 4.7 software package. Electrodes were cycled for 3-5 times in saline solution in order to get stable voltammetric responses before performing the measurements with the wine spirit samples.

\subsubsection{Voltammetric measurements}

Prior to its measurement, samples were firstly diluted in $0.1 \mathrm{M} \mathrm{KCl}$ in order to ensure a good conductivity of the media. In addition, all samples were diluted to the same abv level $\left(\sim 17.4^{\circ}\right)$ to prevent the high level of alcohol in the original samples damaging the sensors and also to avoid that this factor could be a source of discrimination ${ }^{9}$.

Cyclic voltammetry measurements were carried out at room temperature $\left(25^{\circ} \mathrm{C}\right)$ under quiescent conditions and with no other sample pretreatment than its mere dilution with $\mathrm{KCl}$ solution. Potential was swept between $-1.0 \mathrm{~V}$ and $+1.3 \mathrm{~V}$ vs $\mathrm{Ag} / \mathrm{AgCl}$, with a 
scan rate of $100 \mathrm{mV} \cdot \mathrm{s}^{-1}$ and a step potential of $9 \mathrm{mV}$. Apart, all experiments were carried out without performing any physical surface regeneration of the working electrodes; only, an electrochemical cleaning stage between each measurement was done in order to prevent the accumulative effect of impurities on the working electrode surfaces. For this, a conditioning potential of $+1.4 \mathrm{~V}$ was applied during $45 \mathrm{~s}$ after each experiment in a cell containing $25 \mathrm{ml}$ of distilled water ${ }^{6}$.

\subsection{Data processing}

All computations and chemometric processing were carried out by specific routines written by the authors using MATLAB 7.1 (MathWorks, Natick, MA) and its Neural Network Toolbox (v4.0.6). For the qualitative approach Linear Discriminant Analysis (LDA) was used as the modelling tool, while quantitative models were based on the use of Artificial Neural Networks (ANNs). Sigmaplot 2000 (Systat Software Inc, California, USA) was used for graphic representations of data and results.

\section{Results and Discussion}

\subsection{Voltammetric responses}

Examples of the different obtained signals produced by the voltammetric ET array for the different samples of both subsets are shown in Figure 1; additionally, obtained response for all the sensors could be seen in supplementary Figures S1 and S2. Voltammograms on the left correspond to the samples from the first subset, the one with qualification of fresh distillates taste; while the ones on the right correspond to the second subset used to evaluate the use of alternative ageing methods.

\section{$<$ FIGURE 1 $>$}

In the first case, it could be seen how some samples present very similar voltammetric responses in specific regions along the voltammogram; also, that analogous resemblances are repeated along the whole recorded signal, but between different samples. E.g. in Figure 1A it could be seen how the samples A16, A23 and A29 show equivalent anodic response at the region around $1 \mathrm{~V}$; while for the cathodic wave (ca. $0 \mathrm{~V})$, A1 shows differentiated response, but still similar signals are obtained for samples A23 and A29, plus sample A27, which presents the same response in this 
region, but not in the other. Also, differentiated trends are obtained for the different sensors from the ET; e.g. in Figure $1 \mathrm{C}$ it could be seen that only samples A1 and A4 show the same response in the anodic region close to $-1 \mathrm{~V}$; which could be somehow expected given both samples were classified to taste type 1 by the sensory panel. Thus, a huge source of variability and cross-response features are obtained for the different samples with the proposed sensor array, a highly desirable situation for an ET system.

Similarly, a consistent behaviour was obtained for the samples of the second subset (graphs on the right of Figure 1). In this case, it is known that some oxidative signals may be due to the total polyphenolic content of the samples ${ }^{15}$, although as it will be shown later, the discrimination is not due to this fact, and as before different trends are shown along the whole voltammogram.

Hence, as can be seen, different voltammetric responses are obtained for each kind of sensor, with different behaviour for each sample, generating very rich data to be used as the departure point. Nevertheless, to fully exploit all the information obtained from each voltammogram, a compression step is required in order to gain advantages in training time, to avoid redundancy in input data and to obtain a model with better generalization ability ${ }^{17}$. Moreover, main reason is due to the extreme complexity of the considered departure signals that would require instead the use of multiway processing strategies ${ }^{18}$.

In order to reduce the large data set generated for each sample (6 sensors x 512 current values at different polarization potentials), a preprocessing stage employing the Fast Fourier Transform (FFT) was used to compress the original sensors information ${ }^{19}$. In this manner, signals from each voltammogram were reduced down to 16 coefficients without any loss of relevant information, which means a compression ratio of $96.9 \%$.

\subsection{LDA classification models}

After data compression, obtained coefficients were used as inputs in a LDA classification model (one independent model for each of the cases). LDA analysis was done using a stepwise inclusion method which allows to remove the variables that have a lower contribution to the classification model ${ }^{20}$, this strategy tries to find the best subset to perform the prediction task as well as possible, with as few variables as can be. In this way, modelling accuracy and robustness of the final model are improved, as well as its easier understanding. 
On the other hand, given this is a supervised method, classification success was evaluated using leave-one-out cross validation. In this way, each sample is classified by means of the analysis function derived from the other samples (all cases except the case itself). Although results with this strategy sometimes are criticized as overoptimistic, its use is necessary when there is no availability of large sample sets ${ }^{21}$.

\subsubsection{Taste attributes}

The first approach consisted assessing if the ET was capable of discriminating the samples according to its taste attributes in the same manner a sensory panel would do. In this sense, ETs have a large advantage over classical methods given they are based on a biomimetic approach. That is, classical analytical methods require the identification of the concrete species responsible of those sensations; while the ET combines all the extracted chemical information from the array of sensors to generate a model like the human brain would do, not requiring to focus attention to which specific compounds are the responsible for those flavours or tastes, but using a generic signal fingerprint instead. Therefore, samples were first tested by a sensory panel, which classified them into three classes from " 1 " to " 3 ", i.e. from best to worst taste quality. LDA model was built employing their scores as the classes to be distinguished, extracting and using the knowledge from the experts.

\section{$<$ FIGURE 2>}

As can be seen in Figure 2, a clear discrimination for the three qualities of distillates was achieved. Moreover, from the plot it could also be seen that samples are sorted along DF1 according to its taste class, with a complete agreement with the order assigned by the sensory panel.

\section{$<$ TABLE 1 $>$}

Classification results of LDA leave-one-out cross-validation approach are summarized in Table 1. As expected from the LDA plot, all samples were correctly classified according to its taste class. Hence, the classification rate of the samples reached $100 \%$ of accuracy. The efficiency of the classification obtained was also evaluated according to its sensitivity, i.e. the percentage of objects of each class 
identified by the classifier model, and to its specificity, the percentage of objects from different classes correctly rejected by the classifier model. The value of sensitivity, averaged for the classes considered was, $100 \%$, and that of specificity was $100 \%$.

\subsubsection{Ageing method}

As done in the previous case, a new LDA model was built and evaluated using leave-one-out cross validation method, now with the second subset of samples. In this case, the target was to see if the ET was able to distinguish the different uses of oak pieces of wood in the ageing of brandy to reproduce its ageing in new barrels while accelerating the process. Thus, in this case samples were distributed in five groups for the model construction: (I) chips, (II) new barrel, (III) sticks, (IV) blocks and (V) strips.

\section{$<$ FIGURE 3>}

As shown in Figure 3, also in this case, a clear discrimination between the five classes of spirits was achieved, where well established clusters evidence that samples are grouped according to its ageing process. Analyzing more deeply the obtained plot, it could be seen that barrel samples are the more distant from the other clusters, while samples using alternative methods are closer one to each other. Also the distance of each group from barrel cluster could give an idea of how different is the proposed method from the original one. In this sense, some authors claim that a key parameter is the size of the pieces of wood ${ }^{22}$. For example, the two closer variants being chips and sticks, evidently two procedures only distinguished by the size and shape used.

\section{$<$ TABLE 2>}

Again, model accuracy was evaluated employing leave-one-out cross validation, which results are summarized on Table 2. As expected from the LDA plot, nearly all samples were correctly classified, with a classification rate of $97.0 \%$ in terms of accuracy; while sensitivity and specificity values were $80.0 \%$ and $99.1 \%$. It should be noticed that there was only one sample misclassified, which corresponded to the blocks type. This is due to the fact that only one sample from this class was available for the study; therefore, when describing the model class with the leave-one-out cross validation method, the absence of other similar samples prevented its correct 
identification. Thus, when evaluating its class, the pattern recognition method only was able to assign it to the nearest cluster, the strip.

\subsubsection{Vintage identification}

Additionally, given the importance of the vintage to the quality of the obtained product (in the case of wine), and given brandies are obtained from its distillation, the effect of vintage was also studied. In fact, it was examined the existence of any fingerprint in the brandies related to vintage that might be detectable by means of the ET. Hence, employing the samples from the first subset, a new LDA model was built taking into account this parameter. In this case samples were distributed in five groups for the model construction, from 2008 to 2012 vintage.

$<$ FIGURE 4>

As shown in Figure 4, also in this case, a clear discrimination between the different vintages of spirits was achieved, where well established clusters evidence that samples are grouped according to its vintage. Analyzing more deeply the obtained plot, it could be seen that samples from 2012 are the more distant from the other clusters, as could be expected given that are the less aged and mainly separated by DF1; while the rest of samples are closer one to each other and discriminated also thanks to DF2.

\section{$<$ TABLE 3>}

Again, model accuracy was evaluated employing leave-one-out cross validation, which results are summarized on Table 3. As expected from the LDA plot, nearly all samples were correctly classified, with a classification rate of $95.1 \%$ in terms of accuracy; while sensitivity and specificity values were $78.8 \%$ and $98.7 \%$. Again, it should be noticed that there were only two samples misclassified, one of those correspond to a class where only one sample was available for the study which prevented its correct identification.

\subsection{ANN quantitative models}

As a further ability of electronic tongue sensor systems, a quantitative application in order to predict compounds of relevant significance in brandies was also attempted. In 
this case, concentrations of the sought compounds were modelled from the set of voltammetric responses, previously compressed with FFT, by means of an ANN model. In this sense, and depending on the qualitative approach, different compounds were analyzed by classical methods for both subsets to extract additional quantitative analytical information that may complement ET qualitative response if their level could be modelled properly.

In the first case, given the importance to keep under certain levels some undesired compounds, mainly alcohols, responsible of taste defects in the fresh wine distillates ${ }^{23}$, those were quantified by standard procedures employing Gas Chromatography (GC). In the second case, given the importance of polyphenols in the ageing of wine, these were quantified employing the polyphenol index $\mathrm{I}_{280}$ as the quick reference procedure ${ }^{24}$. Then, the objective was to correlate ET response with specific compounds or with general index concentrations. As before, coefficients obtained after FFT analysis were used as inputs of the ANN model, building two different models for each of the subsets.

The first step, prior to building the ANN model, is the selection of the topology of the network. This is a trial-error process that requires a significant effort of optimization due to the difficulties in predicting the optimum ANN configuration in advance, where several parameters (training algorithms, number of hidden layers, transfer functions, etc.) are fine-tuned in order to find the best configuration to optimize the performance of the neural network model ${ }^{17}$. Thus, multiple ANN architectures and topologies were assayed employing Bayesian regularization algorithms. Comparison graphs of predicted vs. expected concentrations for each of the compounds under study were built to check the prediction ability of the ANN.

\subsubsection{Markers of some brandy defects}

In order to provide more relevant information of the samples that compliments its taste category, the identification of undesired compounds was also attempted in a semi-quantitative approach. Hence, much attention was focused to specific compounds known to induce some defects on brandy samples ${ }^{4}$, and its quantification was attempted by means of the ET approach.

Generally, alcohols with more than two carbon atoms, known as higher alcohols and mainly produced during fermentation, and also their esters have intense odours that play a role in wine aromas. At low concentrations, they contribute to its aromatic complexity; while at higher levels, their penetrating odours mask its aromatic finesse ${ }^{23}$. 
Particularly, butan-2-ol, ethyl acetate, acetaldehyde and butan-1-ol were the specific substances under study. Among those, butan-2-ol as such has no sensory impact but is often a marker of bacterial alterations coming from their lees in wines poorly conserved; acetaldehyde is an intermediate compound of the alcoholic fermentation, which concentration can be increased due to several factors such as the addition of $\mathrm{SO}_{2}$; ethyl acetate induces a strange smell (similar to solvent glue) and is generated in sour wine from acetic acid and ethanol.

To estimate presence of these markers, concentrations levels of each of the compounds were divided into specific domains to a three-level scale (i.e. -1 for normal levels, 0 for slight defect and +1 for clear defect) according to food industry

requirements (Table 4) ${ }^{25}$, which values were then modelled employing a fuzzy logic prediction. Although the levels of some specific compounds can be used as markers of some brandy defects, it should be taken into account that they are only markers and not determine final taste category. That is, even that some samples may have relatively high levels of one of those compounds, this does not imply that the sample taste classification will be demoted given its quality could be improved or demoted due to the presence of other compounds not quantified or identified. Some examples could be found in Table S1; e.g. samples A36 and A37 both have low levels for the four considered compounds, and yet assigned to worst taste quality, while opposite situation could be seen for sample A8.

\section{$<$ TABLE $4>$}

In this manner, a semi-quantitative ANN fuzzy model for the ordinal quantification of 4 different compounds was trained employing $75 \%$ of the data, using the remaining $25 \%$ of the data (testing subset) for the evaluation of model's performance employing the k-fold cross-validation method. After some preliminary tests, the final architecture of the ANN model had 96 neurons (6 sensors x 16 coeffs. obtained from the FFT analysis) in the input layer, 6 neurons and tansig transfer function in the hidden layer and four neurons and purelin transfer function in the output layer, providing samples level of the four compounds under study.

As can be seen in Figure 5, where comparison graphs of predicted vs. expected levels for each of the compounds were built, good prediction ability was attained by the model. Although larger dispersion was obtained for a few samples, good trend is 
obtained in general, with fitted regression lines almost indistinguishable from the theoretical ones.

$<$ FIGURE 5 $>$

\subsubsection{Polyphenol index}

After evaluating the total polyphenol content of the spirits using the reference method, it was confirmed that there was no relation between its index value and the type of ageing method employed; and what's more, having nearly the same mean value for all the classes (Figure 6). Hence, it was confirmed that discrimination of samples by the ET was not due to this parameter.

$<$ FIGURE 6>

Nevertheless, capability of the ET system to correctly predict polyphenol index in brandies was evaluated; an approach already tested successfully in the case of wines ${ }^{15}$. For this purpose, a quantitative ANN model was built and optimized. After some preliminary tests, the final architecture of the ANN model had 96 neurons (6 sensors $\mathrm{x}$ 16 coeffs. obtained from the FFT analysis) in the input layer, 6 neurons and tansig transfer function in the hidden layer and one neuron and purelin transfer function in the output layer, providing as output the predicted polyphenol index.

Accuracy of the generated model was evaluated employing a jack-knife method to avoid dependence of predictions from the specific subdivision of data, training the model with $75 \%$ of the data ( 25 samples) and doing its external test with the remaining $25 \%$ (8 samples). In this way, train/test data subdivision was repeated randomly 25 times in order to ensure that model's accuracy is good enough and that ET performance does not depend on the specific subsets used. Then, once all responses from all the constructed models were obtained, predicted values by each model were grouped depending if they were used in the training process or in the testing subset (similarly to k-fold method). Finally, average values for each sample were calculated, allowing us to calculate model uncertainties and obtain unbiased data ${ }^{26}$.

Comparison graphs of predicted vs. expected $\mathrm{I}_{280}$ index were built to check the prediction ability of the ANN (Figure 7). As can be observed, the obtained comparison parameters are close to the ideal values, with intercept near to 0 and slope and 
correlation coefficient around 1, meaning that there are no significant differences between the values predicted by the ANN model and those expected and provided by the reference method.

$<$ FIGURE 7>

\section{Conclusions}

Electronic Tongues have proved to be a useful analytical tool able to provide information for either the detection of the use of alternative ageing practices such as the use of pieces of wood or to asses a quality index of brandy samples according to skilled sensory panel results. Moreover, it has also allowed the semi-quantitative detection of undesired compounds above certain levels or the quantitative prediction of polyphenol content according to $\mathrm{I}_{280}$ method.

In this sense, ET represent an alternative to traditional methods with advantages over those such as being a fast-response and low-cost method for its characterization, really suitable for screening analysis. Furthermore, given its biomimetic behaviour, it presents a straightforward solution to the absence of the knowledge about which compounds are primarily responsible for the brandy style. What is more, the proposed ET system has been able to extract knowledge from the expertise gathered in the sensory panel and build correspondence with the signal fingerprint provided by the sensor array. And, this is accomplished overcoming the problems found with conventional analytical techniques such as spectroscopy and chromatography which application to such tasks is really hindered by the fact that responsible compounds may not have been identified yet.

\section{Acknowledgments}

Financial support for this work was provided by Spanish Ministry of Science and Innovation, MCINN (Madrid) trough project CTQ2010-17099 and by program ICREA Academia from Generalitat de Catalunya. X. Cetó thanks the support of Dept. d'Innovació, Universitats i Empresa de la Generalitat de Catalunya for the predoctoral grant. 


\section{References}

1. P. Ciosek and W. Wroblewski, Analyst, 2007, 132, 963-978.

2. M. del Valle, Electroanal., 2010, 22, 1539-1555.

3. Y. Vlasov, A. Legin, A. Rudnitskaya, C. Di Natale and A. D'Amico, Pure Appl. Chem., 2005, 77, 1965-1983.

4. A. J. Buglass, D. J. Caven-Quantrill, N. J. Fuller, J. A. Hudson, C. G. Lee, S. H. Lee and M. McKay, Handbook of Alcoholic Beverages: Technical, Analytical and Nutritional Aspects, John Wiley \& Sons, Ltd, 2011.

5. J. Zeravik, A. Hlavacek, K. Lacina and P. Skladal, Electroanal., 2009, 21, 25092520.

6. X. Cetó, J. M. Gutiérrez, L. Moreno-Barón, S. Alegret and M. del Valle, Electroanal., 2011, 23, 72-78.

7. A. Rudnitskaya, E. Polshin, D. Kirsanov, J. Lammertyn, B. Nicolai, D. Saison, F. R. Delvaux, F. Delvaux and A. Legin, Anal. Chim. Acta, 2009, 646, 111-118.

8. P. Ciosek and W. Wróblewski, Talanta, 2006, 69, 1156-1161.

9. W. Novakowski, M. Bertotti and T. R. L. C. Paixão, Microchem. J., 2011, 99, 145-151.

10. K. Toko, Meas. Sci. Technol., 1998, 9, 1919.

11. J. M. Gutiérrez, L. Moreno-Barón, M. I. Pividori, S. Alegret and M. del Valle, Microchim. Acta, 2010, 169, 261-268.

12. M. Gay, C. Apetrei, I. Nevares, M. del Alamo, J. Zurro, N. Prieto, J. A. De Saja and M. L. Rodriguez-Mendez, Electrochim. Acta, 2010, 55, 6782-6788.

13. A. Rudnitskaya, E. Lochner, M. Lambrechts and A. Legin, Application of the Electronic Tongue (ET) to the analysis of brandy, St.Petersburg, Russia, 2007.

14. A. Legin, A. Rudnitskaya, B. Seleznev and Y. Vlasov, Anal. Chim. Acta, 2005, 534, 129-135.

15. X. Cetó, J. M. Gutiérrez, M. Gutiérrez, F. Céspedes, J. Capdevila, S. Mínguez, C. Jiménez-Jorquera and M. del Valle, Anal. Chim. Acta, 2012, 732, 172-179.

16. F. Céspedes, E. Martínez-Fàbregas and S. Alegret, TrAC-Trend. Anal. Chem., 1996, 15, 296-304.

17. F. Despagne and D. Luc Massart, Analyst, 1998, 123, 157R-178R.

18. D. Ebrahimi, E. Chow, J. J. Gooding and D. B. Hibbert, Analyst, 2008, 133, 1090-1096. 
19. X. Cetó, F. Céspedes and M. del Valle, Talanta, (in press).

20. R. A. Johnson and D. W. Wichein, Applied multivariate statistical analysis, Prentice-Hall, Upper Saddle River, N.J., 2007.

21. D. Kirsanov, O. Mednova, V. Vietoris, P. A. Kilmartin and A. Legin, Talanta, 2012, 90, 109-116.

22. M. Del Alamo Sanza and I. Nevares Domínguez, Anal. Chim. Acta, 2006, 563, 255-263.

23. P. Ribéreau-Gayon, Y. Glories, A. Maujean and D. Dubourdieu, Handbook of Enology, Volume 2, The Chemistry of Wine Stabilization and Treatments, WileyBlackwell, London, 2000.

24. A. L. Waterhouse, in Current Protocols in Food Analytical Chemistry, John Wiley \& Sons, Inc., 2001.

25. J. Sourisseau, Bull. Union Physiciens, 2002, 96, 881-892.

26. J. Shao and D. Tu, The Jackknife and bootstrap, Springer, New York, 1995. 
Table 1. Confusion matrix built according wine spirits taste category obtained using LDA model and leave-one-out cross validation.

\begin{tabular}{cccc}
\hline Found & Taste 1 & Taste 2 & Taste 3 \\
\hline Expected & 14 & 0 & 0 \\
Taste 1 & 0 & 10 & 0 \\
Taste 2 & 0 & 0 & 17 \\
Taste 3 & Percentage Classification & $100 \%$ \\
\hline
\end{tabular}


Table 2. Confusion matrix built according wine spirits ageing method obtained using LDA model and leave-one-out cross validation.

\begin{tabular}{cccccc}
\hline Found & \multirow{2}{*}{ Chips } & New Barrel & Sticks & Blocks & Strips \\
\hline Chips & 15 & 0 & 0 & 0 & 0 \\
New barrel & 0 & 3 & 0 & 0 & 0 \\
Sticks & 0 & 0 & 4 & 0 & 0 \\
Blocks* & 0 & 0 & 0 & 0 & 1 \\
Strips & 0 & 0 & 0 & 0 & 10 \\
\hline \multicolumn{5}{c}{ Percentage Classification } \\
\hline
\end{tabular}

*Only one sample was available 
Table 3. Confusion matrix built according wine spirits vintage obtained using LDA model and leave-one-out cross validation.

\begin{tabular}{|c|c|c|c|c|c|}
\hline Expected & 2008 & 2009 & 2010 & 2011 & 2012 \\
\hline $2008 *$ & 0 & 0 & 0 & 1 & 0 \\
\hline 2009 & 0 & 12 & 0 & 0 & 0 \\
\hline 2010 & 0 & 0 & 9 & 0 & 0 \\
\hline 2011 & 1 & 0 & 0 & 16 & 0 \\
\hline 2012 & 0 & 0 & 0 & 0 & 2 \\
\hline \multicolumn{5}{|c|}{ Percentage Classification } & $95.1 \%$ \\
\hline
\end{tabular}

*Only one sample was available 
Table 4. Concentration ranges, expressed in $\mathrm{mg} \cdot \mathrm{L}^{-1}$, used for the division of samples into the three-level classes used for the semi-quantitative approach.

\begin{tabular}{ccccc} 
& butan-2-ol & ethyl acetate & acetaldehyde & butan-1-ol \\
\hline-1 & $<1$ & $<350$ & $<30$ & $<3$ \\
0 & $1 \leq \mathrm{X}<2.5$ & $350 \leq \mathrm{X}<450$ & $30 \leq \mathrm{X}<50$ & $3 \leq \mathrm{X}<6.6$ \\
+1 & $\geq 2.5$ & $\geq 450$ & $\geq 50$ & $\geq 6.6$ \\
\hline
\end{tabular}




\section{FIGURE CAPTIONS}

Figure 1. Example of the different obtained voltammograms with the sensor array: (A, C) graphite-epoxy, (B, D) platinum nanoparticle and (E, F) copper nanoparticle. Graphs on the left correspond to fresh distillates of different quality from the first subset (section 2.2.1), while the ones on the left correspond to spirits produced by different ageing methods subset (section 2.2.2). Detailed information of the samples is available on the supplementary information.

Figure 2. Score plot of the functions obtained after LDA analysis of the wine spirits, according to its taste category. As can be seen, clear discrimination is obtained for the different spirits: $(\bullet)$ taste $1,(\boldsymbol{\Delta})$ taste 2 and $(\square)$ taste 3 . Also, the centroid of each class is plotted $(\star)$.

Figure 3. Score plot of the functions obtained after LDA analysis of the wine spirits, according to the ageing method used. As can be seen, clear discrimination is obtained

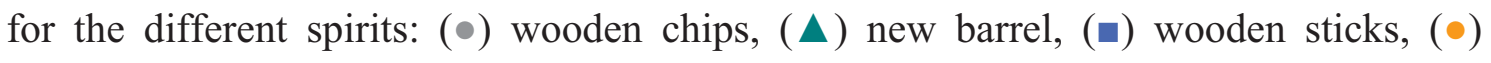
wooden blocks and $(\diamond)$ wooden strips. Also, the centroid of each class is plotted $(\star)$.

Figure 4. Score plot of the functions obtained after LDA analysis of the wine spirits, according to its vintage. As can be seen, clear discrimination is obtained for the

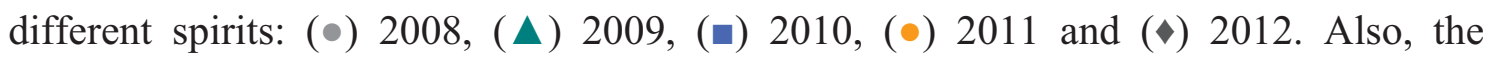
centroid of each class is plotted $(\star)$.

Figure 5. Modelling ability of the optimized FFT-ANN for the semi-quantitative prediction of four compounds used as markers of some taste defects. Sets adjustments of obtained vs. expected values for the testing subset is shown. Dashed line corresponds to theoretical diagonal line.

Figure 6. Polyphenol content of each class as measured by $I_{280}$ method. Average content and its standard deviation are shown for each class, as well as its maximum and minimum. 
Figure 7. Modelling ability of the optimized FFT-ANN for the prediction of $\mathrm{I}_{280}$ index. Sets adjustments of obtained vs. expected values for the testing subset is shown. Dashed line corresponds to theoretical diagonal line. Results provided correspond to the average of the values obtained for each sample after 25 repeated calculations, done with random division of samples for train/test subsets each time. Uncertainties calculated at the $95 \%$ confidence level. 

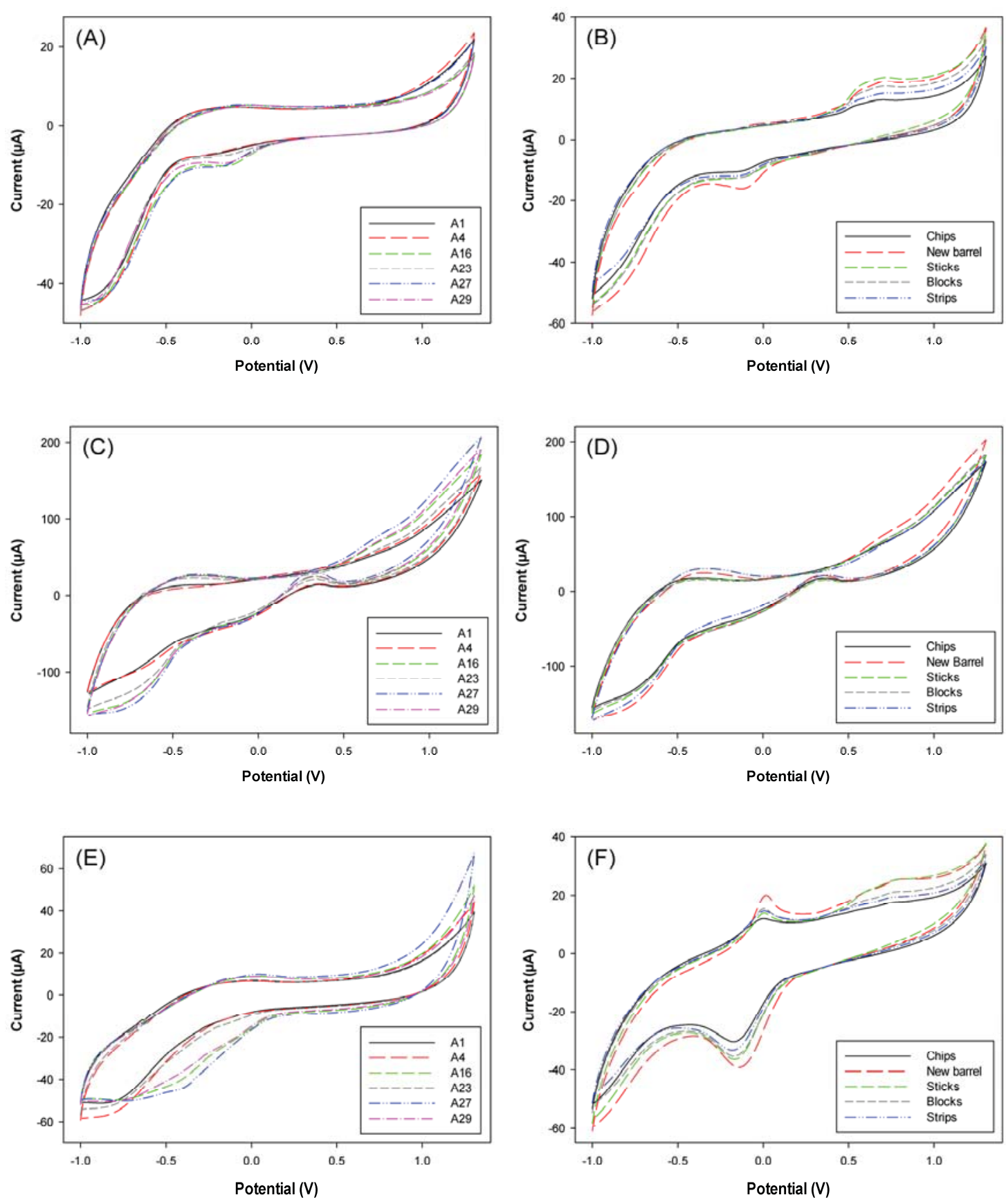

Figure 1 


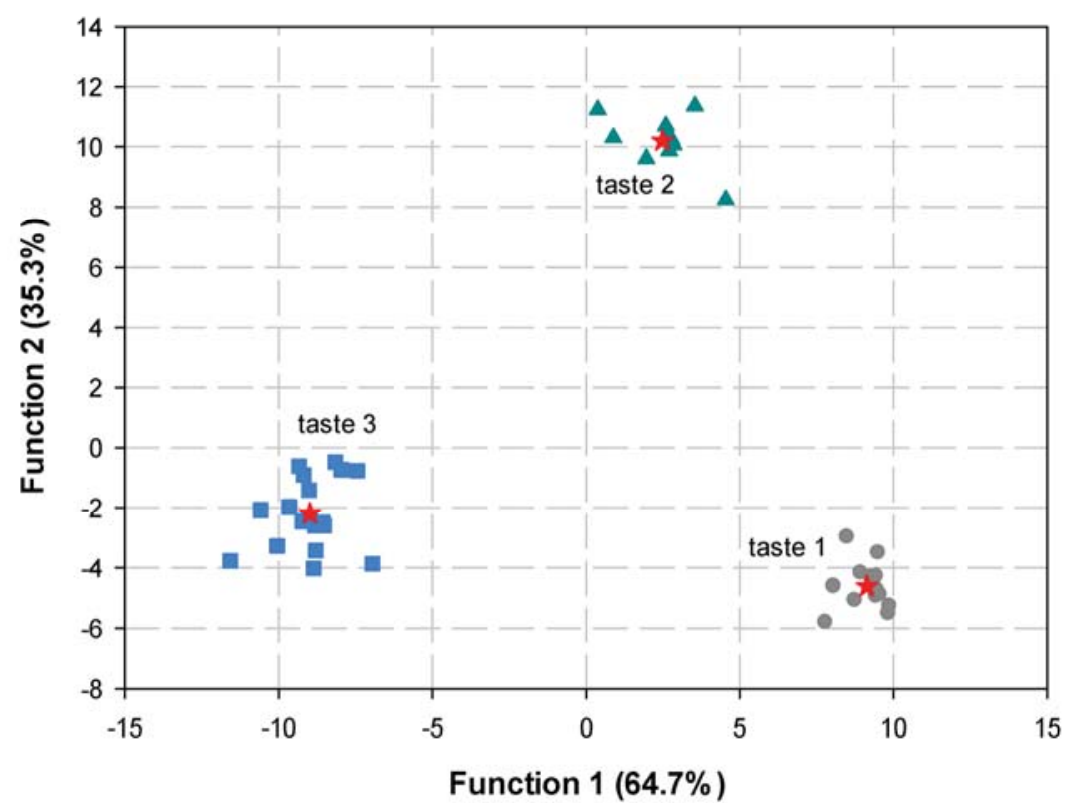

Figure 2 


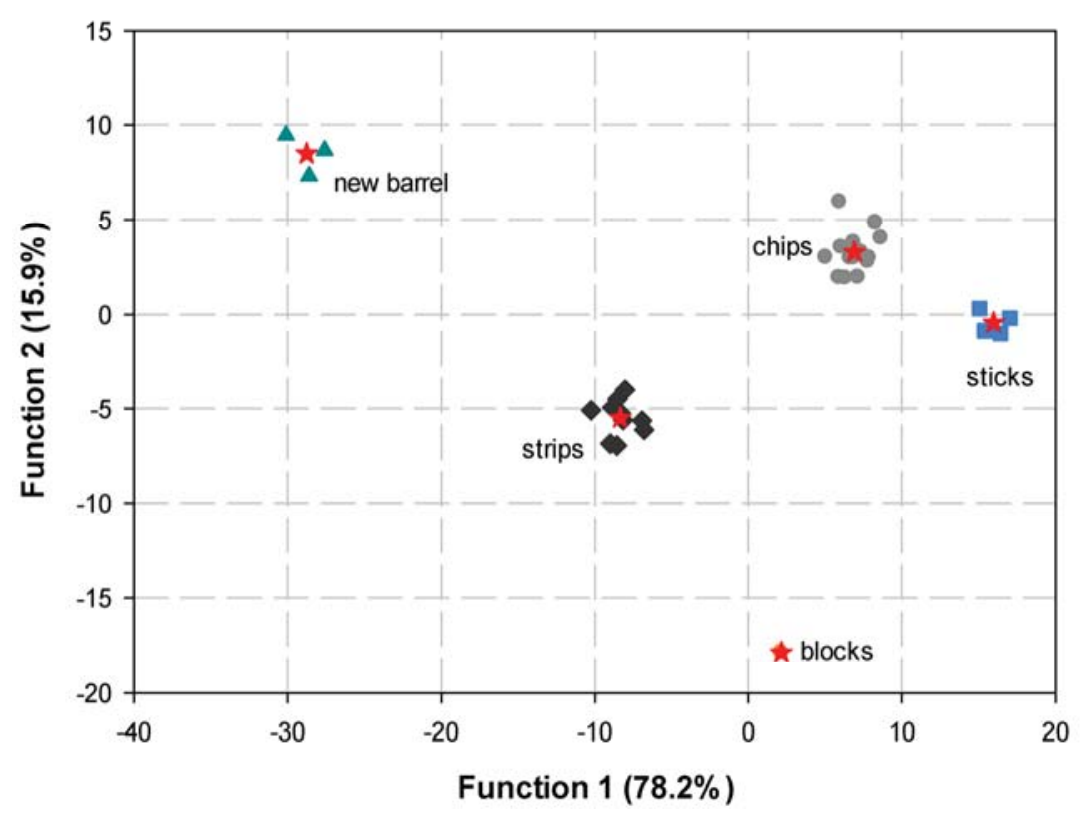

Figure 3 


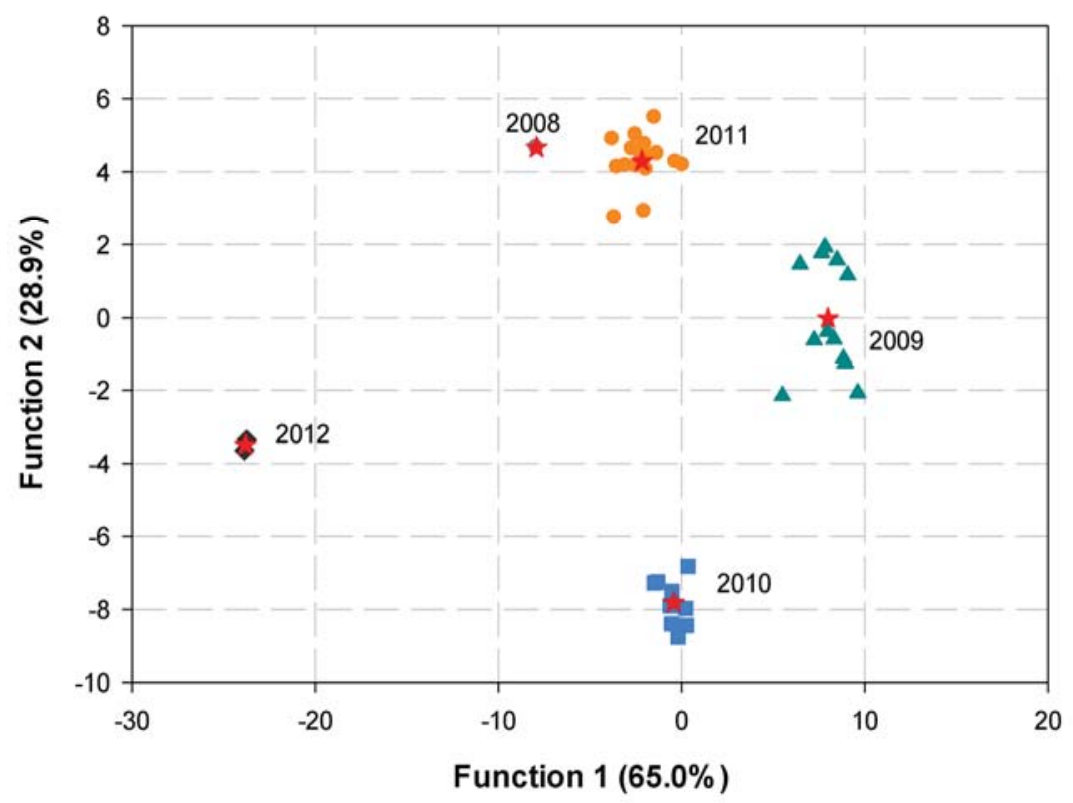

Figure 4 

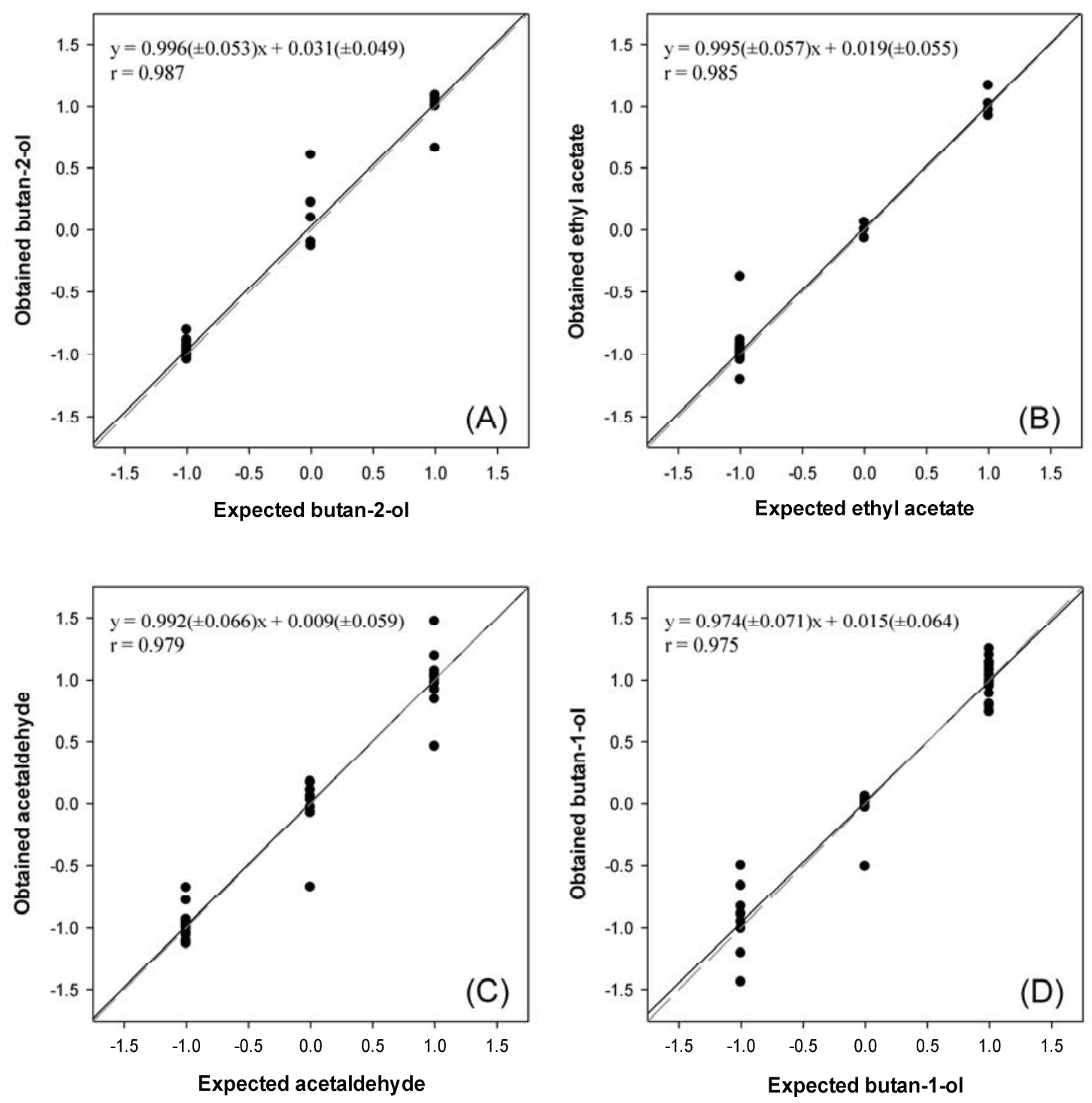

Figure 5 


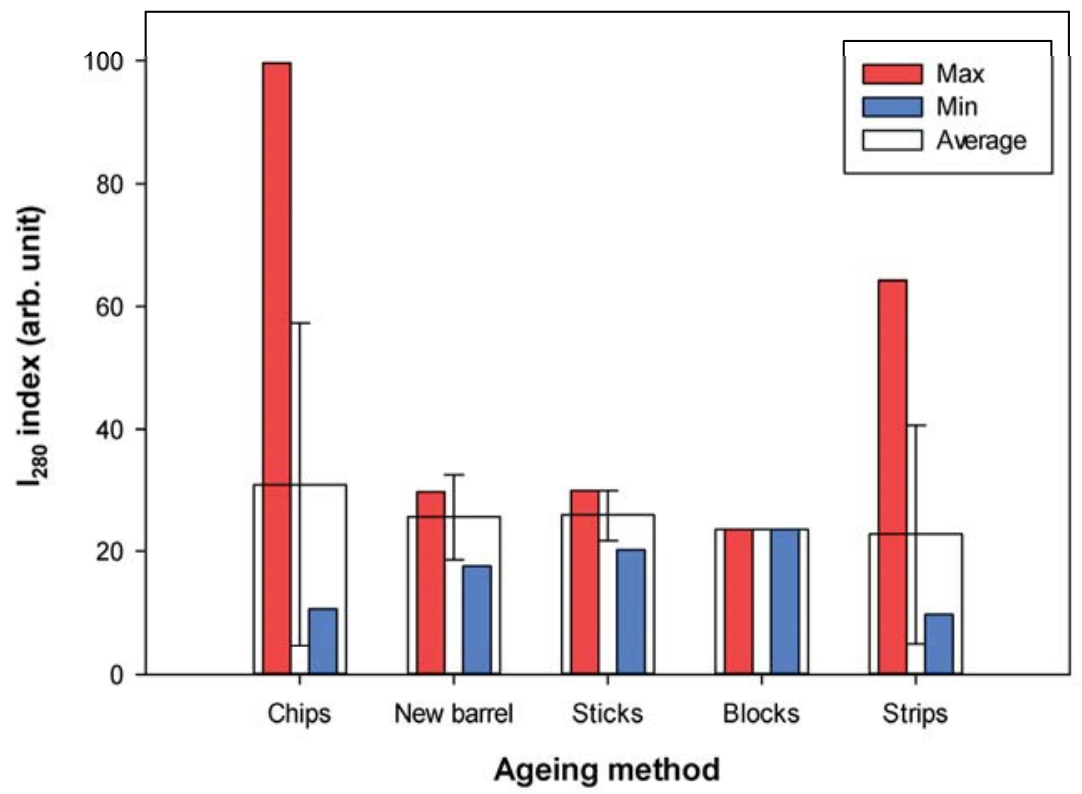

Figure 6 


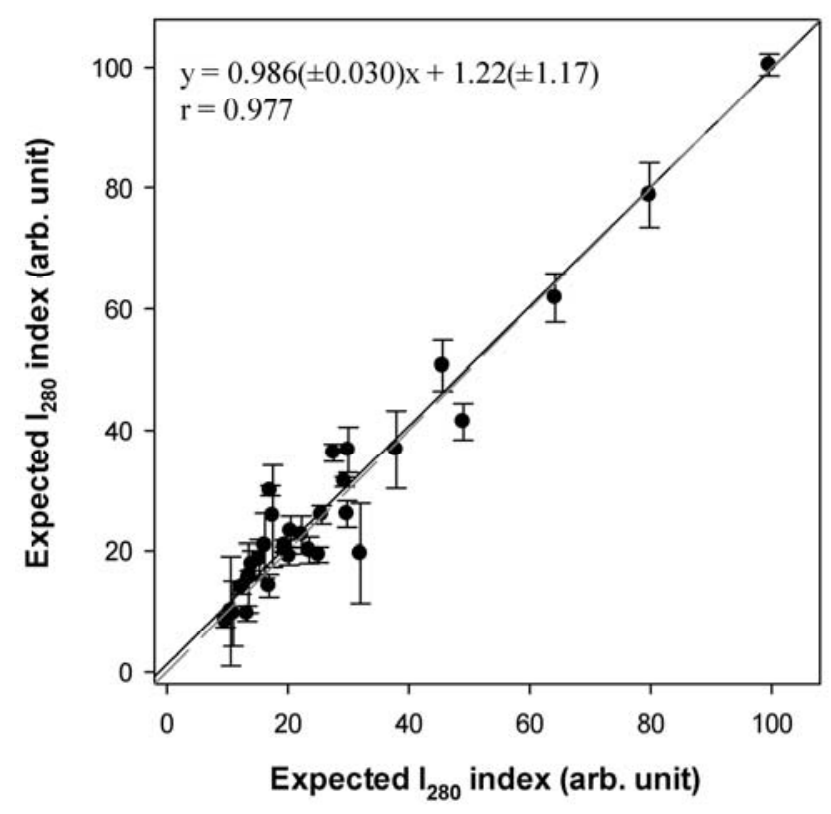

Figure 7 